\title{
Thermohaline convection and metallic fingers in polluted stars
}

\author{
Sylvie Vauclair \\ Laboratoire d'Astrophysique, Observatoire Midi-Pyrénées, 14 avenue Edouard Belin, 31400 \\ Toulouse, France \\ email:sylvie.vauclair@obs-mip.fr
}

\begin{abstract}
When a layer of heavy matter is above lighter matter in a star, the inverse $\mu$-gradient may lead to thermohaline (or double-diffusive) convection. This has been studied in the past for helium-rich atmospheres, but it may also occur for metal-rich layers. It has recently been studied for the accretion of hydrogen poor material onto the host stars of exoplanets. These stars present a metallicity excess compared to stars in which no planets have been detected. However, the reason for this excess is still a subject of debate. It may be primordial or the result of accretion, or both. In this last case, thermohaline convection may lead to "metallic fingers" which partially dilute the accreted matter inside the star. Such an effect can also be important in the chemically peculiar A stars in which metals accumulate in the atmospheric layers.
\end{abstract}

Keywords. Accretion, convection, diffusion, stars: abundances

\section{Introduction}

Spectroscopic observations of stars sometimes give evidence of overmetallicity compared to "normal" stars. In the chemically peculiar A stars, heavy elements are supposed to accumulate in the atmosphere due to radiative support. Solar type stars around which planets have been detected, compared with stars without planets clearly show a metallicity excess of a factor two on the average, while the individual $[\mathrm{Fe} / \mathrm{H}]$ values lie between -0.3 and +0.4 (Santos et al. 2003 and references therein). The proposed possible explanations for this behavior are either that the star and its planets formed out of an already metal-rich cloud (primordial hypothesis) or that a non-negligible number of planets fell into the star during the formation process (accretion hypothesis). In the last case, overmetallic matter would be above matter with a normal composition, as in peculiar A stars.

Vauclair (2004) showed that in this case we must account for the convective instability induced by inverse $\mu$-gradients, or thermohaline convection (also called "double-diffusive convection"). If metals accumulate in stellar outer layers, they will not stay there but will be partially diluted downwards in "metallic fingers" similar to the "salt fingers" observed in the ocean. The exact abundances of the metals which may remain in the stellar outer layers depend on parameters like the size and depth of the metallic fingers, which cannot be precisely constrained in the framework of our present knowledge. Here we show the importance of this process, which should be taken into account in the computations, and studied more precisely with the help of numerical simulations.

\section{Thermohaline convection}

Thermohaline convection is a well known process in oceanography. Warm salty layers on the top of cool unsalted ones rapidly diffuse downwards even in the presence of stabilizing temperature gradients. When a blob is displaced downwards, it continues moving 


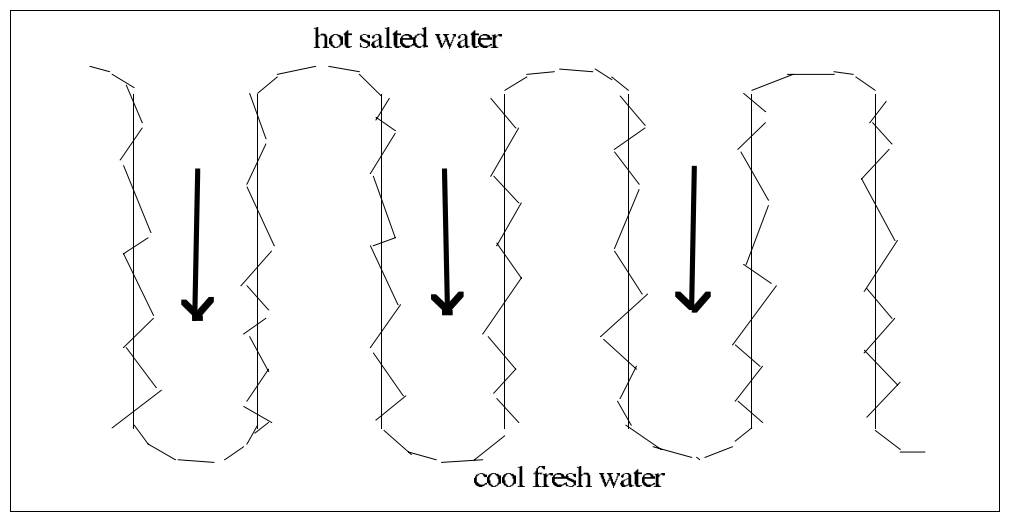

Figure 1. Schematic drawing of salt fingers: for hot salted water on top of cool fresh water, the salinity gradient is destabilizing while the temperature gradient is stabilizing; the medium should be stable except that heat diffuses more quickly than salt between the fingers and the interfinger medium. Because of the difference in diffusivities, a blob which begins to fall continues this motion until it mixes completely with the surroundings. This creates a special kind of convection which is well known in oceanography. A similar case occurs in stars for layers with inverse $\mu$-gradients when the temperature gradients are stabilizing. The broken lines at the edges of the fingers are drawn as symbols of the partial mixing which occurs there, due to shear flow instabilities. This mixing is difficult to evaluate, which is the basic reason for the uncertainties in the computations of the final abundances.

downward due to its excess density compared with the surroundings, but at the same time that it is hotter contradicts this tendancy. When the salt gradient is large compared to the thermal gradient, salted water normally mixes down until the two effects compensate. Then thermohaline convection begins. While the medium is marginally stable, salted blobs fall like fingers while the unsalted matter goes upward. This process is commonly known as "salt fingers" (Stern 1960, Kato 1966, Veronis 1965, Turner 1973, Turner \& Veronis 2000, Gargett \& Ruddick 2003). The reason why the medium is still unstable is due to the different diffusivities of heat and salt (for this reason it is also called "double-diffusive convection"). A warm salted blob falling in cool fresh water sees its temperature decrease before the salt has time to diffuse out. The blob goes on falling due to its density until it mixes with the surroundings (Figure 1).

The condition for the salt fingers to develop is related to the density variations induced by the temperature and the salinity perturbations. Two important characteristic numbers are defined :

- the density anomaly ratio

$$
R_{\rho}=\alpha \nabla T / \beta \nabla S
$$

where $\alpha=-\left(\frac{1}{\rho} \frac{\partial \rho}{\partial T}\right)_{S, P}$ and $\beta=\left(\frac{1}{\rho} \frac{\partial \rho}{\partial S}\right)_{T, P}$ while $\nabla T$ and $\nabla S$ are the average temperature and salinity gradients in the considered zone

- the so-called "Lewis number"

$$
\tau=\kappa_{S} / \kappa_{T}=\tau_{T} / \tau_{S}
$$

where $\kappa_{S}$ and $\kappa_{T}$ are the saline and thermal diffusivities while $\tau_{S}$ and $\tau_{T}$ are the saline and thermal diffusion time scales.

The density gradient is unstable and overturns into dynamical convection for $R_{\rho}<1$ while the salt fingers grow for $R_{\rho} \geqslant 1$. On the other hand they cannot form if $R_{\rho}$ is larger than the ratio of the thermal to saline diffusivities $\tau^{-1}$ as in this case the salinity difference between the blobs and the surroundings is not large enough to overcome 
buoyancy (Huppert \& Manins 1973, Gough \& Toomre 1982, Kunze 2003). Salt fingers can grow if the following condition is satisfied :

$$
1 \leqslant R_{\rho} \leqslant \tau^{-1}
$$

\section{The stellar case}

Thermohaline convection may occur in stellar radiative zones when a layer with a larger mean molecular weight sits on top of layers with smaller ones (Kato 1966, Spiegel 1969, Ulrich 1972, Kippenhahn et al. 1980). In this case $\nabla_{\mu}=\operatorname{dln} \mu /$ dln $P$ plays the role of the salinity gradient while the difference $\nabla_{\text {ad }}-\nabla$ (where $\nabla_{\text {ad }}$ and $\nabla$ are the usual adiabatic and local (radiative) gradients $\operatorname{dln} T / \mathrm{d} \ln P$ ) plays the role of the temperature gradient. Different situations may occur according to the respective values of these gradients. When $\nabla_{\text {ad }}$ is smaller than $\nabla_{\text {rad }}$, the temperature gradient is unstable against convection (Schwarszchild criterion) which corresponds to warm water below cool water in oceanography. In the opposite case the temperature gradient is stable but the medium can become convectively unstable for inverse $\mu$ gradients (heavy matter above lighter one).

When the destabilizing effect of the $\mu$ gradient is larger than the stabilizing effect of the temperature gradients, the medium is unstable against dynamical convection: this is the so-called "Ledoux criterium". Namely the medium is unstable if the following condition is satisfied : :

$$
\nabla_{\text {crit }}=\frac{\phi}{\delta} \nabla_{\mu}+\nabla_{\text {ad }}-\nabla<0
$$

where $\phi=(\partial \ln \rho / \partial \ln \mu)$ and $\delta=(\partial \ln \rho / \partial \ln T)$.

When this situation occurs, convection first takes place on a dynamical time scale and the $\mu$ enriched matter mixes down with the surroundings until $\nabla_{\text {crit }}$ vanishes.

When the stability condition is achieved, we could suppose that the medium remains stable. When a blob begins to fall, it is heavier than its surroundings and would like to go on falling, but in this situation the temperature gradients act in the inverse direction and should be able to prevent it from falling. However, this is when thermohaline convection begins because, as for the salted case in the ocean, the heat diffuses more rapidly out of the fingers than the particles (Figure 1). In the process of the falling blob, heat diffuses between the blob and its surroundings so that it becomes less efficient to support it against its extra-weight. This leads to thermohaline or double-diffusive convection which mixes matter as a "secular process", namely on a thermal time scale (short compared to the stellar lifetime!).

Such an effect has previously been studied for stars with a helium-rich accreted layer (Kippenhahn et al. 1980). It was also invoked for helium-rich stars in which helium is supposed to accumulate due to diffusion in a stellar wind (as proposed by Vauclair 1975) and for roAp stars in case some helium accumulation occurs (Vauclair et al. 1991).

The study of thermohaline mixing in stars is far from trivial. Detailed comparisons of numerical simulations and laboratory experiments in the water case have recently been published (Gargett \& Ruddick 2003), but the stellar case may be different as mixing then occurs in a compressible stratified fluid. Comparing the stellar case with the water case, we can guess that metallic fingers will form if the following condition is verified :

$$
1 \leqslant\left|\frac{\delta\left(\nabla_{\mathrm{ad}}-\nabla\right)}{\phi\left(\nabla_{\mu}\right)}\right| \leqslant \tau^{-1}
$$




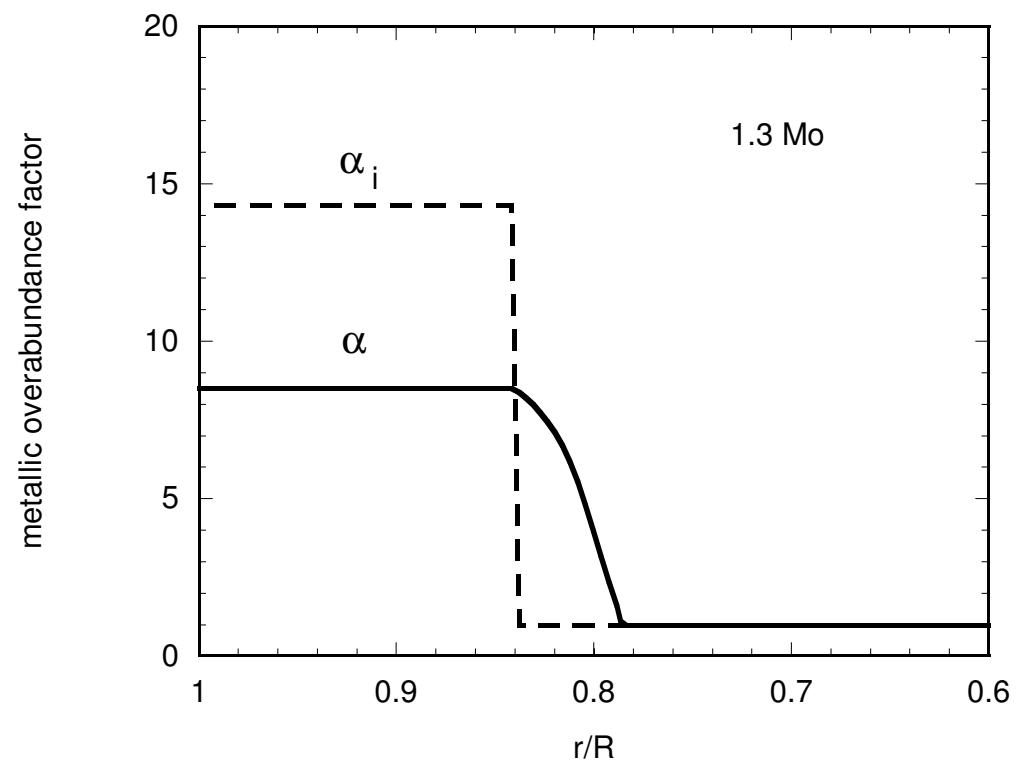

Figure 2. This figure presents the metallicity profiles in a $1.3 M_{\odot}$ star, in the case of accretion, before and after dynamical convection. The ordinate $\alpha$ stands for the overmetallic ratio, i.e., the ratio of the global abundances of metals compared to the initial (solar) one. Thermohaline convection begins after this phase.

with $\tau=D_{\mu} / D_{T}=\tau_{T} / \tau_{\mu}$ where $D_{T}$ and $D_{\mu}$ are the thermal and molecular diffusion coefficients while $\tau_{T}$ and $\tau_{\mu}$ are the corresponding time scales.

As will be discussed below, the most important unknown about the fate of the fingers concerns the local mixing which occurs between the fingers and the interfinger regions. This diffusion process is basically due to shear flow instabilities at the boundary of the fingers (Figure 1) and depends on their velocity, which in turn depends on the diffusion process. Moreover, the whole process may be perturbated in case of other kinds of turbulence, like rotation-induced mixing. Only numerical simulations can help these diffculties.

\section{The fate of overmetallic layers in stars}

Vauclair (2004) studied Main-Sequence solar type stars which would have accreted hydrogen-poor material at the beginning of their lifetime. I assumed, for simplicity, that the accretion occurred in a very short time scale compared to stellar evolution. I then studied the fate of the accreted metals and chose the examples of $1.1 M_{\odot}$ and $1.3 M_{\odot}$ stars. I first computed the depth at which metal-enriched material is diluted and the actual overabundance ratio in the convective zone, when it reaches the marginal equilibrium phase, which is obtained when $\nabla_{\text {crit }}$ vanishes (Equation 3.1) Then I used the formalism proposed by Kippenhahn et al. (1980) to evaluate the effect of thermohaline convection.

The whole picture may be described as follows: blobs of metal enriched matter begin to fall from the convective zone and exchange heat and heavy elements with their surroundings, Chemicals diffuse more slowly than heat, so that the blobs continue falling until they are completely disrupted, thereby creating finger shapes. The most efficient process for element diffusion out of the blobs is the shear flow instabilities at the edge of the fingers. As the falling matter undergoes friction with the rising matter, turbulence 


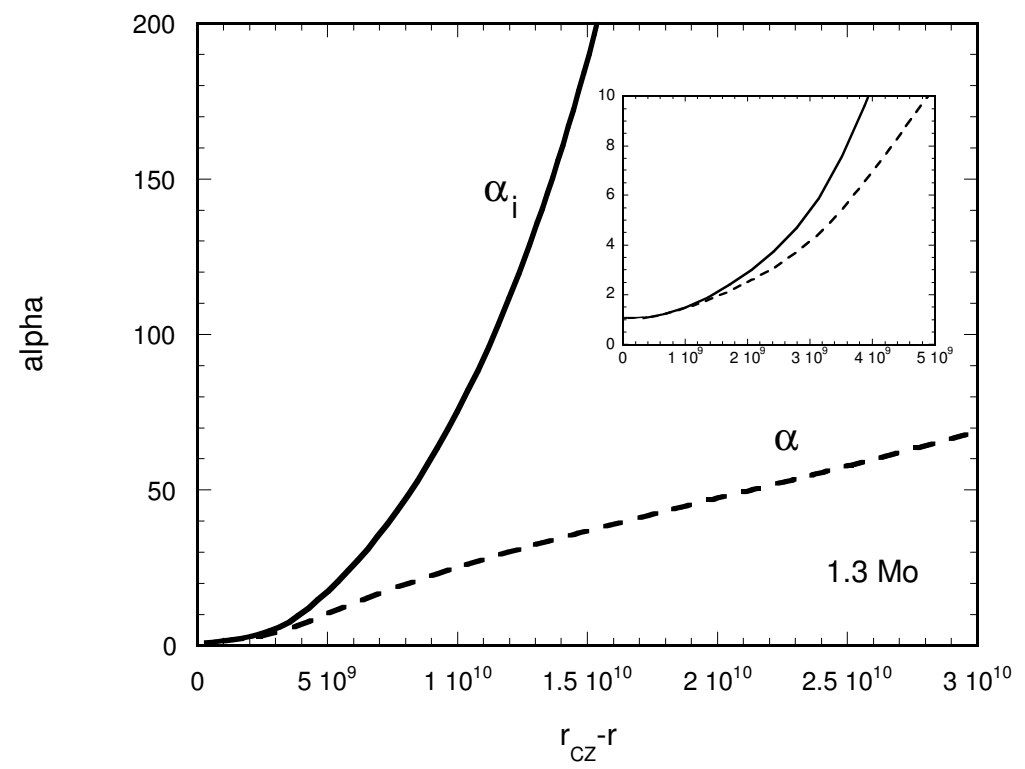

Figure 3. This graph displays, for a $1.3 M_{\odot}$ star, the overmetallic ratio at the end of the dynamical phase, according to the original one. It also shows the depth at which the overmetallic matter is mixed after this phase. Namely, for a given initial overmetallic ratio $\alpha_{i}$, the depth (below the convective zone) at which it will be mixed is obtained in abscissa from the $\alpha_{i}$ curve, and the resulting overmetallic ratio is then obtained from the $\alpha$ curve. Thermohaline convection occurs after this phase, and the final overmetallic ratio is difficult to evaluate.

occurs and mixes part of the fingers with their surroundings, on a horizontal length scale which is a fraction $\epsilon$ of the horizontal size of the blobs. The blobs disappear when they have travelled a distance long enough for this mixing to disrupt them completely. The remaining metal abundances in the convective zone are related to the depth of the fingers, which, as we have seen, is difficult to evaluate.

Although approximative, the computations show that metallic matter in the stellar outer layers should not stay there. It is expected to be partially mixed due to the dynamical convection and then should diffuse due thermohaline convection.

In chemically peculiar A stars, the destabilizing effect due to metal accumulation in the outer layers may be compensated by the stabilizing effect of the helium-gradient induced by downward diffusion. It should, however, be studied in details as it may modify in a non-negligible way the final abundance anomalies.

This type of convection should be studied with numerical simulations, as it may have important effects anytime overmetallic layers are above matter with a normal chemical composition.

\section{References}

Gargett, A., Ruddick, B. 2003, ed., Progress in Oceanography, vol 56, issues 3-4, pages 381-570, special issues on "Double diffusion in Oceanography",

Gough, D.O., Toomre, J., 1982, J. Fluid Mech, 125, 75

Huppert, H.E., Manins, P.C. 1973, Deep-Sea Research, 20, 315

Kato, S., 1966, PASJ, 18, 374

Kunze, E. 2003, Progress in Oceanography, 56, 399

Kippenhahn, R., Ruschenplatt, G., Thomas, H.C. 1980, A\&A, 91, 175

Santos, N.C., Israelian, G., Mayor, M., Rebolo, R., Udry, S. 2003, A\&A, 398, 363 
Spiegel, E. 1969, Comments on Ap and Space Phys, 1, 57

Stern, M.E., 1960, Tellus, 12, 2

Turner, J.S., 1973, Buoyancy effects in fluids, Cambridge University Press

Turner, J.S., Veronis, G. 2000, Journal Fluid Dynamics, 405, 269

Ulrich, R.K., 1972, ApJ, 172, 165

Vauclair, S., 1975, A\&A, 45, 233

Vauclair, S., 2004, ApJ, 605, 874

Vauclair, S., Dolez,N., Gough, D.O. 1991, A\&A, 252, 618

Veronis, G.J., 1965, Marine Res., 21, 1

\section{Discussion}

ShiBAHAshi: Thermohaline convection in a star that you discussed seems to be the nonradial thermal instability. If so, I think the instability condition $\left(\nabla_{\mu}<0\right.$ and $\left.\nabla_{\text {ad }}>0\right)$ shoud be the necessary condition but not the sufficient one, and whether or not the instability occurs indeed is not determined locally but by the gloval analysis (as in the case of overstability due to $\nabla-\nabla_{\text {ad }}>0$ and $\nabla_{\mu}>0$ ). Is your conclusion based on the local stability analysis?

VAUCLAIR: The instability criterion is determined locally but the extent to which the fingers develop and the efficiency of the resulting mixing depends on the situation in subjacent layers: this is a nonlocal treatment.

NoELS: I agree with you that with a gradient of helium the thermohaline convection can be very efficient. However, in the case of an accumlation of iron for example, don't you think that the change in $\mu$ is too small that it cannot trigger such a mixing?

VAUCLAIR: Of course metals contribute much less than helium to the mean molecular weight. But in some cases their accumulation can be large enough for this process to occur. It is the case for accretion, for example. In the case of element diffusion, generally helium falls down while metals go up. We may expect that the overall $\mu$-gradient remains stabilizing. However, if metals accumulate in a region where the helium profile is flat, this process may occur and limit the metal abundance.

PRESTON: In your salt finger example what causes the convection to continue after the heavy overlying material has sunk?

VAUCLAIR: It continue if the process which forces the heavy matter to go up continues. The difficulty is to compute correctly the competitive process. For example in helium rich stars, helium accumulates due to the stellar wind but falls down due to fingers. The observations show that a factor of two overabundance remains but it is difficult to compute this result theoretically. 